\title{
Left ventricular improvement due to allogeneic CB-MNCs transplantation in a chronic heart failure six-years after myocardial infarction
}

\author{
Li Zhu ${ }^{1}$, Aihuan Yue ${ }^{2}$, Zhongbao Ruan ${ }^{1}$, Yigang Yin ${ }^{1}$, \\ Ruzhu Wang ${ }^{1}$, Yin Ren ${ }^{1}$, Gecai Chen ${ }^{1}$ \\ ${ }^{1}$ Department of Cardiology, Taizhou People's Hospital, Taizhou, Jiangsu Province, China \\ ${ }^{2}$ Jiangsu Province Beike Stem Cell Bank, Taizhou, Jiangsu Province, China
}

\section{Introduction}

Recent studies have shown that bone marrow mononuclear cell (BM-MNC) therapy improves exercise capacity in patients with more advanced chronic heart failure (HF) [1]. However, few studies have explored the safety profile and efficacy of allogeneic umbilical cord blood mononuclear cells (CB-MNCs) administered intravenously to patients with $\mathrm{CHF}$.

The umbilical cord blood was taken from healthy full-term and naturally delivered newborns after written informed consent were obtained from their mothers and other family members. The study protocol was reviewed and approved by the hospital review board and ethics committee of Taizhou People's Hospital (Taizhou, China). Also, the study protocol was performed in compliance with current Good Clinical Practice standards.

\section{Case presentation}

A 68-year-old man was sent to a nearby community hospital because of chest pain and was diagnosed as myocardial infarction (MI) in September 2008. The main symptoms of acute MI were as follows: (1) bosom frowsty chest pain; (2) dynamic changes of MI, as shown in electrocardiogram (ECG); (3) peak creatine kinase-MB level exceeding $100 \mathrm{U} / \mathrm{L}$ (upper limit of normal < $24 \mathrm{U} / \mathrm{L}$ ). According to conditions of hospital treatment, the patient was treated with thrombolytic therapy according to ACC/AHA about ST-segment elevation
MI treatment guidelines [2, 3]. After thrombolysis, the patient had ventricular fibrillation, $\mathrm{HF}$ and pulmonary infection. The patient was transferred to our hospital for coronary angiography and stent implantation (implantation site: anterior descending branch) in September 2008 and heart function was class III-IV according to ACC heart function classification standard. But after percutaneous coronary intervention treatment, patient continued to report chest tightness, shortness of breath, even when he slightly stretched himself.

Drug therapy, coronary angiography and stent implantation were given. Drug therapy: enteric-coated aspirin $100 \mathrm{mg}$ once per night, clopidogrel $75 \mathrm{mg}$ once each morning, atorvastatin once per night, angiotensin converting enzyme inhibitor once each morning, low molecular weight heparin morning and evening ( $12 \mathrm{~h}$ apart) subcutaneously. In addition to low molecular weight heparin for 7 days, others were administered continuously. Every morning furosemide $20 \mathrm{mg}$ and spironolactone $20 \mathrm{mg}$ were administered until the operation. Operation: Coronary angiography suggested that the middle left anterior descending artery (LAD) stenosis was nearly $99 \%$ and the first diagonal branch stenosis 70\%. A Cypher (Cordis) was implantated in the LAD. The patient showed severe HF. Nine months later, an alternative approach with CB-MNCs was applied to the patient.

The content of CD34+ was $0.28 \%, 0.32 \%$, $0.25 \%$, and $0.26 \%$. The cell viability of $\mathrm{CB}-\mathrm{MNCs}$ was $98 \%, 96 \%, 99 \%$, and $98 \%$. Without cryopreservation and thawing, $2.0 \times 10^{7}$ allogeneic $\mathrm{CB}-\mathrm{MNCs}$

Address for correspondence: Dr. Gecai Chen, 399 Hailing Road, Taizhou People’s Hospital, Taizhou, Jiangsu Province, China, e-mail: chengecai@163.com

Received: 18.05.2016 Accepted: 20.10.2016 


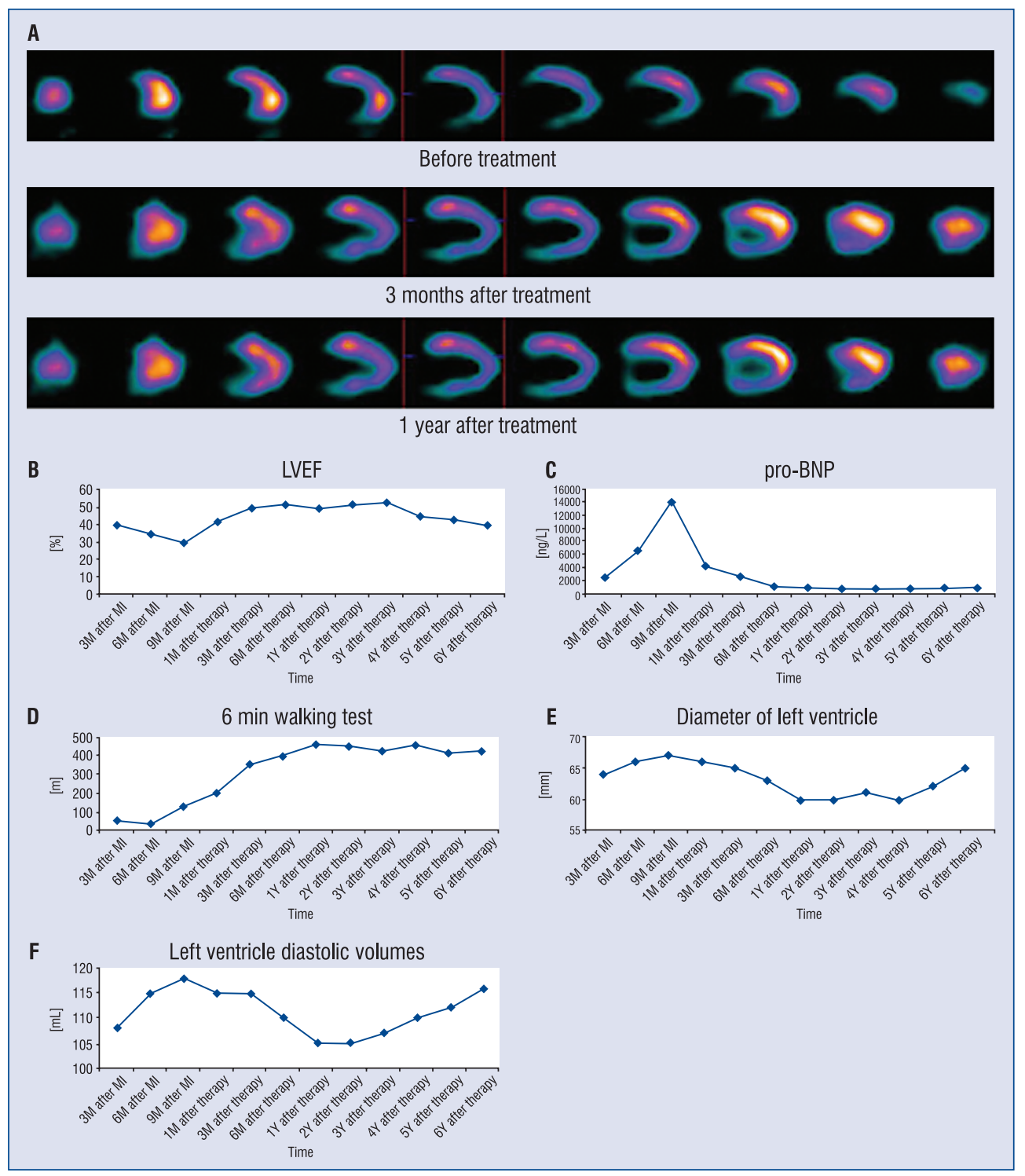

Figure 1. Parameters tracking before and after treatment; $\mathbf{A}$. The infarct region was determined by single-photon emission computed tomography after myocardial infarction (MI), 3 months and 1 year post-transplantation. Compared to before and after treatment the infarct size was considerably reduced by $11 \%$; $\mathbf{B}$. The left ventricular ejection fraction (LVEF) from 3 months after MI to 6 years after therapy; C. The serum level of N-terminal pro-B-type natriuretic peptide (NT-proBNP) reached a peak $(14,130 \mathrm{pg} / \mathrm{mL})$ about 9 months after the onset of symptoms. After cord blood mononuclear cells (CB-MNC) therapy, the serum level of NT-proBNP was maintained at a lower level during the follow-up period; D. The patient's activity improved significantly after CB-MNC therapy. After therapy plus exercise, the patient could walk more than $400 \mathrm{~m}$ in 6 min during the follow-up period; E. The diameters of left ventricle was continuously monitored since it could reflect the size of the heart; $\mathbf{F}$. The left ventricle diastolic volumes reduced 1 month after treatment and rebounded slightly 3 years after treatment; $\mathrm{M}-$ months; $\mathrm{Y}-$ years.

were resuspended in $30 \mathrm{~mL}$ of $0.9 \%$ saline. Before infusion, $5 \mathrm{mg}$ dexamethasone and $25 \mathrm{mg}$ phenergan were added to prevent response every time. CB-MNCs were intravenously injected. The patient received four treatments once a week. All adverse events were monitored, particularly during cell administration. Twenty-four-hour ECG recordings were performed $24-48 \mathrm{~h}$ after cell administration. 


\section{In-hospital patient monitoring and post-discharge follow-up}

Three months after intravenous CB-MNC transplantation, the left ventricular ejection fraction (LVEF) improved significantly by $20 \%$. The initial improvement in the cardiac function was maintained even after 6 years (Fig. 1B). The infarct size considerably reduced by $11 \%$, which was determined by single-photon emission computed tomography after MI and 3 months post-transplantation. Compared to 3 months after treatment, the infarct size was not significantly changed 1 year post-transplantation (Fig. 1A).

The serum level of N-terminal pro-B-type natriuretic peptide (NT-proBNP) reached a peak $(14,130 \mathrm{pg} / \mathrm{mL}$, reference range: $<75$ years old, $<250 \mathrm{pg} / \mathrm{mL}$ ) about 9 months after the onset of symptoms. A high level of NT-proBNP may reflect an acute phase reaction to the extent and severity of the initial ischemic insult. After treatment, the serum level of NT-proBNP was maintained at a lower level ( $<900 \mathrm{pg} / \mathrm{mL})$ (Fig. 1C).

The patient's activity improved significantly after CB-MNC therapy. After treatment and exercise, the patient could walk more than $400 \mathrm{~m}$ in 6 min during the follow-up period (Fig. 1D). The diameters of left ventricle and it's volumes are shown in Figure $1 \mathrm{E}$ and $1 \mathrm{~F}$ and the size of heart is an essential parameter for patients with $\mathrm{HF}$.

\section{Discussion}

This study assessed LVEF using echocardiography. The patient showed a significant increase in LVEF 3 months after therapy. The most fundamental objective was to reduce the infarct area, which caused HF. CB-MNCs therapy is likely to save the stunning myocardium before myocardial necrosis and fibrous connective tissue replacement. The patient with chest tightness, shortness of breath symptoms improved significantly and exercise tolerance had increased compared to previous levels. The outcomes were consistent with those reported earlier [4].

After CB-MNC therapy, the serum level of NT-proBNP showed a sharp decline and was maintained at a lower level. There may have been a minor placebo effect on the 6 -min walk test. The result of the 6 -min walk test and the serum level of NT-proBNP were negatively correlated 6 months after therapy. The heart function of the patient was basically maintained at class II-III 3 months after therapy according to Canadian Cardiovascular Society patient grading. Overall, an improvement in the left ventricular cardiac function and a reduction in the infarct size were induced by CB-MNCs in patients with chronic HF.

\section{Conclusions}

Left ventricular function improvement indicated that allogeneic CB-MNC transplantation is safe and may be effective in patients with $\mathrm{CHF}$. However, the role of allogeneic CB-MNCs in the treatment of chronic HF needs to be further investigated.

\section{Acknowledgements}

The investigators would like to thank the patient who agreed to report on this case.

Conflict of interest: None declared

\section{References}

1. Honold J, Fischer-Rasokat U, Seeger FH, et al. Impact of intracoronary reinfusion of bone marrow-derived mononuclear progenitor cells on cardiopulmonary exercise capacity in patients with chronic postinfarction heart failure. Clin Res Cardiol. 2013; 102(9): 619-625, doi: 10.1007/s00392-013-0574-1, indexed in Pubmed: 23612920.

2. Krumholz H, Anderson J, Bachelder B, et al. ACC/AHA 2008 Performance Measures for Adults With ST-Elevation and Non-ST-Elevation Myocardial Infarction. Circulation. 2008; 118: 2596-2648, doi: 10.1161/CIRCULATIONAHA.108.191099, indexed in Pubmed: 19001027.

3. Giugliano RP, Braunwald E. 2004 ACC/AHA guideline for the management of patients with STEMI: the implications for clinicians. Nat Clin Pract Cardiovasc Med. 2005; 2(3): 114-115, doi: 10.1038/ncpcardio0135, indexed in Pubmed: 16265435.

4. Yousef M, Schannwell CM, Köstering M, et al. The BALANCE Study: clinical benefit and long-term outcome after intracoronary autologous bone marrow cell transplantation in patients with acute myocardial infarction. J Am Coll Cardiol. 2009; 53(24): 2262-2269, doi: 10.1016/j.jacc.2009.02.051, indexed in Pubmed: 19520249. 